\title{
Using the Polygraph Validation Test (PVT) in Solving Conflicted Polygraph Results and Confirming Deliberate Distortions by Examinees
}

Key words: Polygraph Validation Test, Polygraph Verification Test, Integrated Zone Comparison Test, countermeasures, augmentations

\section{Introduction}

The Polygraph Validation Test (PVT) is an innovative testing method originally invented to help to protect against false positive outcomes and assist the examiner in overcoming denials from individuals later verified as deceptive. This paper will further explore its use in solving conflicted polygraph results and confirming deliberate distortions by examinees in their original examinations as an attempt at countermeasures or augmentations.

\footnotetext{
*tuvia@liecatcher.com
} 
Countermeasures are deliberate attempts by a deceptive examinee to distort the polygraph data in an attempt to create a truthful or inconclusive outcome. Augmentations are deliberate attempts by a truthful examinee to distort the polygraph data in an attempt to ensure a correct truthful outcome. While examiners are often able to identify deliberate distortions, they cannot distinguish if these distortions were motivated by someone trying to beat the test or someone trying to help the examiner make the proper decision.

The PVT is administered in a Peak of Tension (POT) format, where the issue of each question concerns a possible cause of an examinee failing, or why they may have had a problem in their original comparison question test (CQT).

\section{Method}

In our profession, we sometimes encounter a client who is not fully confident with our results or the fact that our result does not serve his or her goals. For example, a theft occurred in a secured place and one of the guards is suspected of being involved. The security company sends the guard to be tested and the result is deceptive. This result may result in the security company assuming responsibility for the theft, or worse, even losing the contract. Which is why the security company sends the guard to be retested by another polygraph firm. The new firm for whatever reason fails to consult the first polygraphers. They run a new examination, and surprisingly the result is non-deceptive. Now the question which of the results is correct arises?

In September 2015, I was invited to conduct a large quantity of tests in another country. I do not speak its native language nor do I know any examiner who does. Due to the large quantity of tests, I brought another female examiner to participate in the project.

I decided to use the Integrated Zone Comparison Technique (IZCT) with four (4) relevant questions for the first CQT. The sequence was:

Chart I: IR1 SCR IR4 C5 R6 C8 R9 C11 R12 C14 R15 13

Chart II: IR1 C5 R15 C8 R6 C11 R9 C14 R15 SCR 13

Chart III: IR1 SCR IR4 R12 C5 R15 C8 R6 C11 R9 C14 13

If needed Chart IV was added.

The client invited his employees to take a polygraph test. After an employee arrived, a representative of the client debriefed him or her and received a verbal consent to 
take the test (all examinees were aware of the issue being investigated). After a short period of time, the employee entered the examination room, and a pre-test interview was conducted. The test questions were then reviewed with the examinee, and the examinee signed an authorisation agreement to take the test.

Two instruments were used: a Limestone - Paragon by me, and a Lafayette LX4000 by the other examiner. The tests were conducted at two sites, and the examinees were divided into three subgroups. Experienced employees, trained but inexperienced employees, and employees still in training.

After starting the project we encountered the following problems:

1. The native language of the examinees was other than English.

2. The examinees' English vocabulary was mainly related to the line of work.

4. Some of the examinees had not used the English language for a long time.

5. There were cultural differences between the examinees and the examiner which could affect the development and strength of the comparison questions.

6. What will the effect of one examiner being female be?

7. The examinees know each other, which causes leaking of information concerning the test procedure and the comparison questions (as creative as we can be, there is a limited number of possibilities).

8. Countermeasures are learnt from the Internet and the staff may teach one another.

The Company's policy was to give a second chance to every examinee who fails the test. Unlike at school, university or other similar exams, where - should you fail an exam, you need to practice or study, and then you should pass the re-examination easily, the lie in a polygraph exam cannot be changed.

The client's demand was to conduct a re-examination on all those who failed the initial test with us, as well as some re-examinations on employees who had failed the test with a different polygraph provider.

At this point we needed to find a way to show and explain conflicted results or verify a deceptive result in the first test.

On arrival to the re-examinations the examinees were informed that they had not passed the initial test and were instructed to cooperate with the examiner, to be truthful and to try to resolve the problem.

When entering the second test, all examinees were informed by the examiner that there were going to be two exams conducted on that day. The first was to be similar 
to the first test, with questions adjusted only if needed, and the second one was to find out the reason for a negative result in either the initial or the re-examination.

In total, we had fifty-one (51) re-examinations. Twenty-one (21) of the examinees neither gave explanation for their reactions nor made any admissions. Six (6) gave some explanation. Four (4) offered confessions. Seven (7) said that they had a problem understanding English. Three (3) examinees attempted active countermeasures.

Let me start with two cases involving countermeasures:

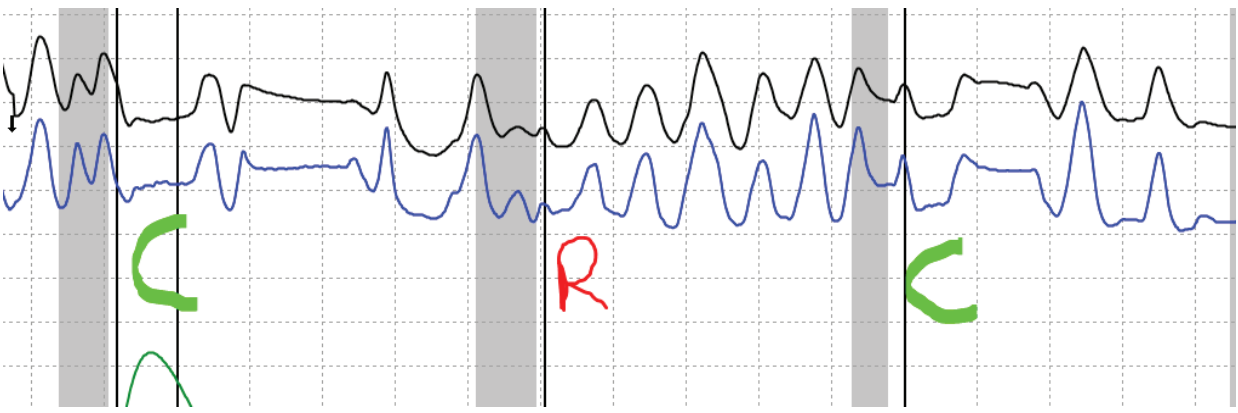

Case A

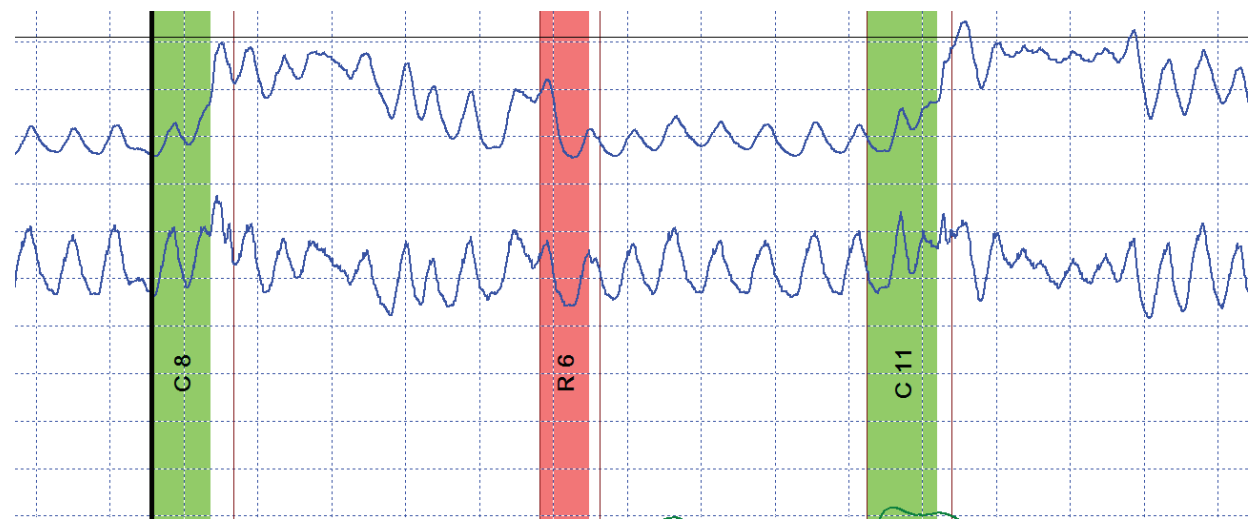

\section{Case B}

Since we had two examiners, the decision was made that the re-examination and the PVT would not be done by the original examiner in order to provide the examinee with a fair and unbiased examination. Before the re-examination both the examinees in question were told the following: 'I saw that you tried to influence the result of the test. Please don't try to help yourself as it will cause the opposite outcome'. The 
examinee in Case A admitted googling polygraph, but denied applying countermeasures. However, the data on his charts on the re-examination were totally different:

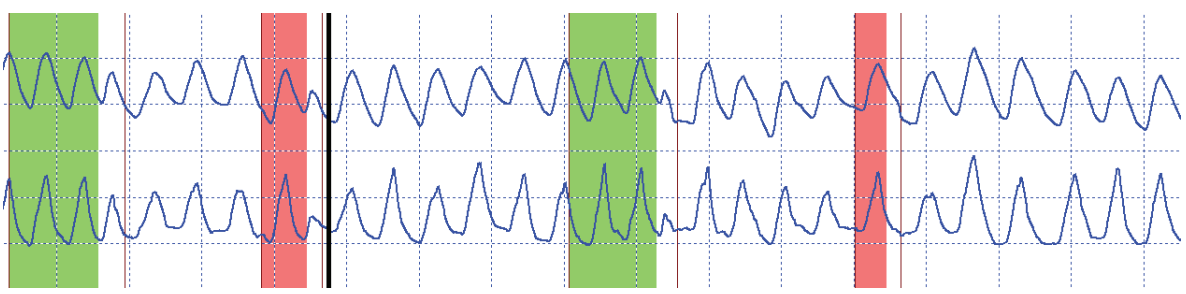

After arriving at a non-deceptive result, a PVT was administered. In the PVT, question C4 referred to the Comparison Questions in the original test, and R5 referred to the Relevant target issue. We found that the examinee's most salient question drawing his psychological set were C4 (classic POT reaction - decrease to point of deception then increase) 'Did you lie to any of the personal questions?' and C6, 'Did you try to influence the result of the exam?' Therefore I believe that the examinee wanted to make sure he would be found truthful.

The examinee in Case B questioned us as to what was observed in the original charts that led us to believe he attempted countermeasures. He was given no answer.

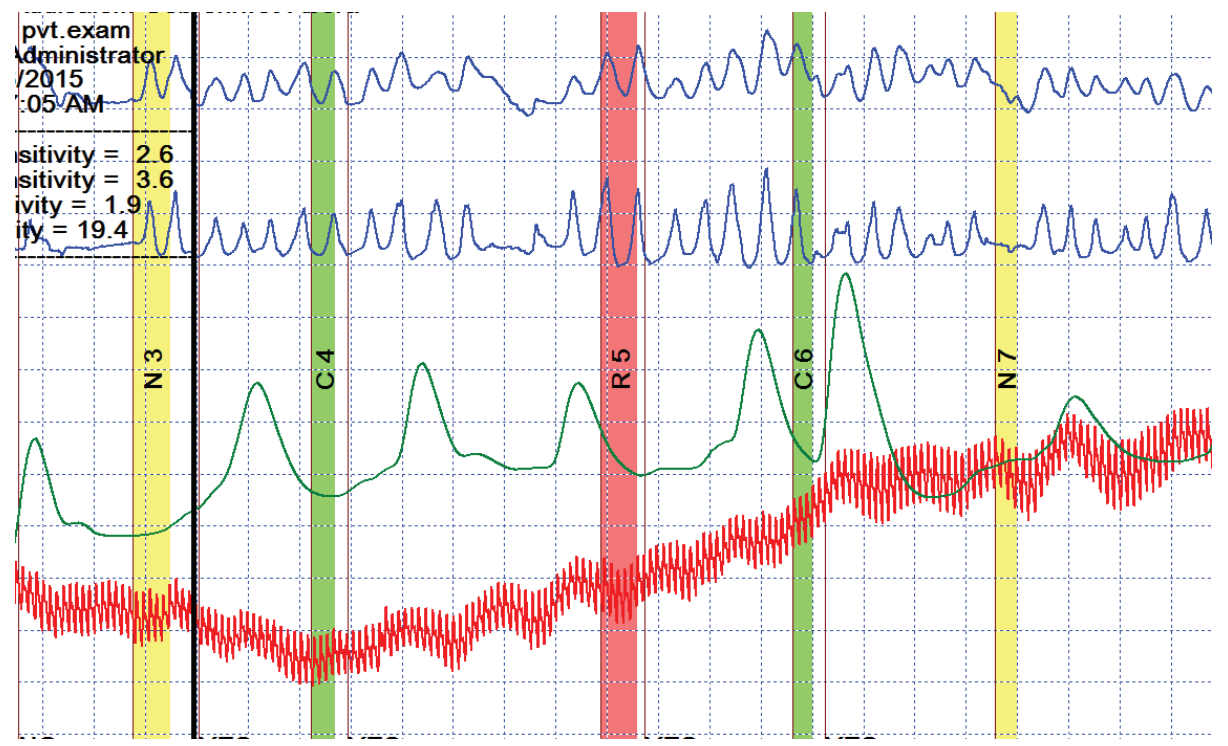




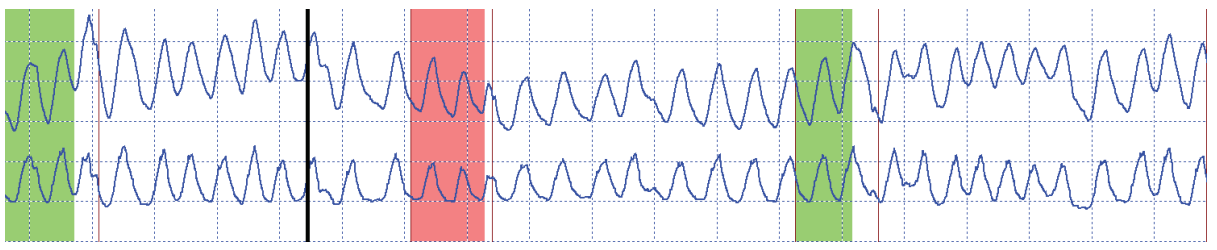

His PVT is presented below:

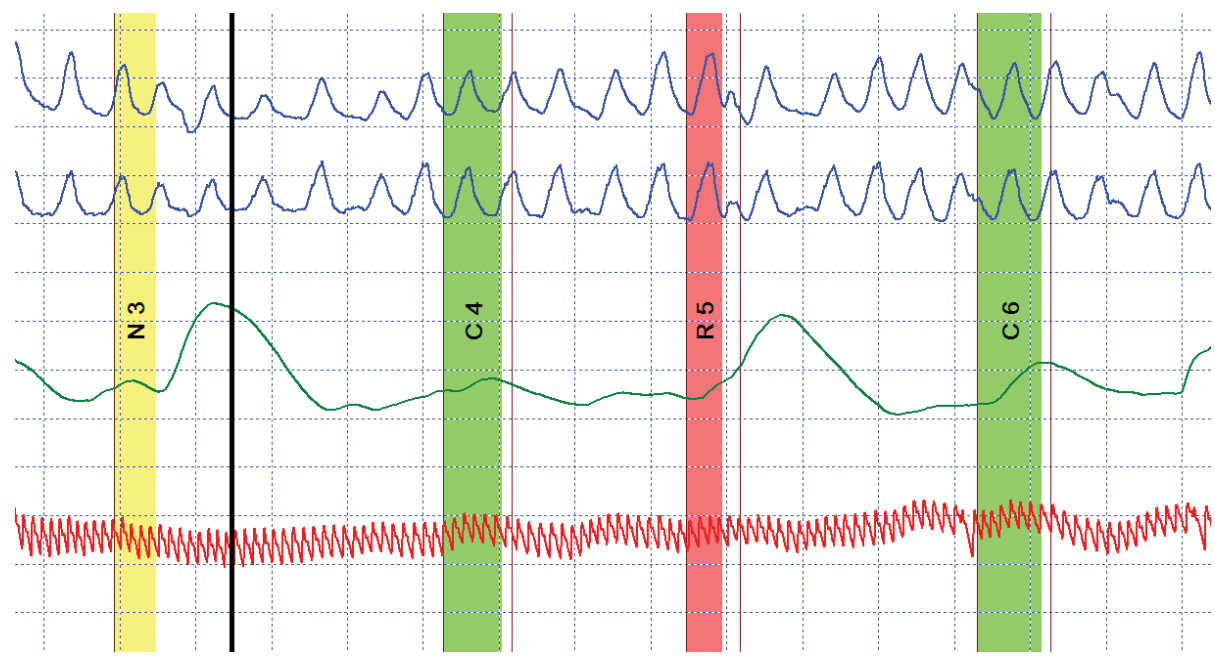

The examinee ceased attempting countermeasures and showed significant reactions to R5 'Did you lie to the target issue?' and N3 'Did you try to influence the result of the test?' Actually, the PVT appeared to confuse the examinee as he did not know what to do or where to attempt distortions.

In the first case, we were able to conclude we had deliberate distortions (augmentations) by a truthful person trying to help us make the proper decision, while the second examinee attempted deliberate distortions (countermeasures) in an attempt to beat the test.

As regards the examinees who gave no explanation, the second test showed deception again. There was no change from the original results! As explained before, to make sure that no unexpected issue affected the result (cultural difference, weak comparison question, language problems, etc.) we conducted a PVT. In all deceptive cases except one, without any explanation, the PVT showed that the examinee focused his attention on the question 'because you lied regarding involvement in the target issue?' In the one exception, the examinee failed to show significant responses to any of the questions. 


\section{PVT - deferent types of reactions}
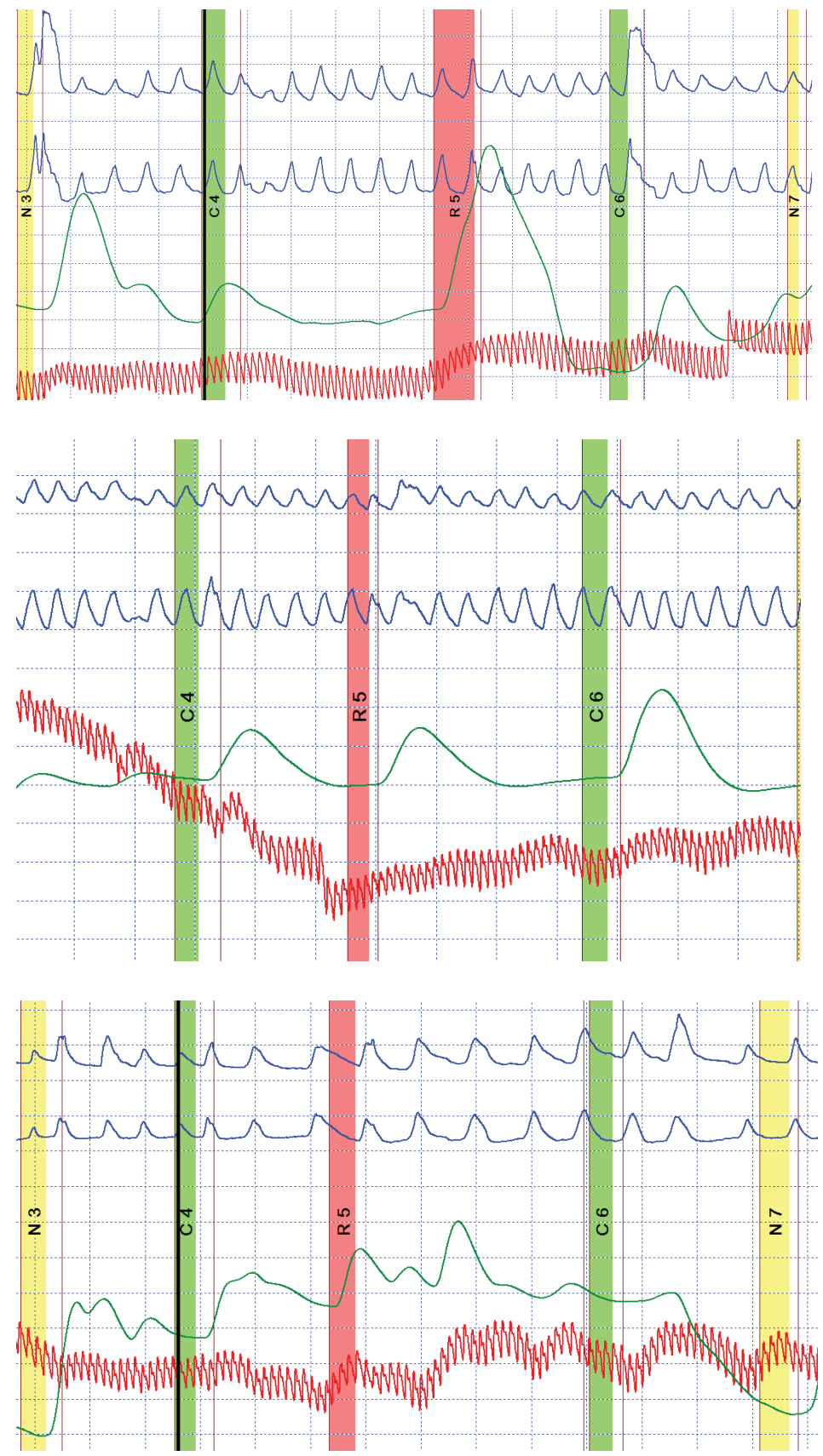
Regarding those who gave confessions or explanations, they clearly showed significant reactions indicating a non-deceptive result in their PVT.
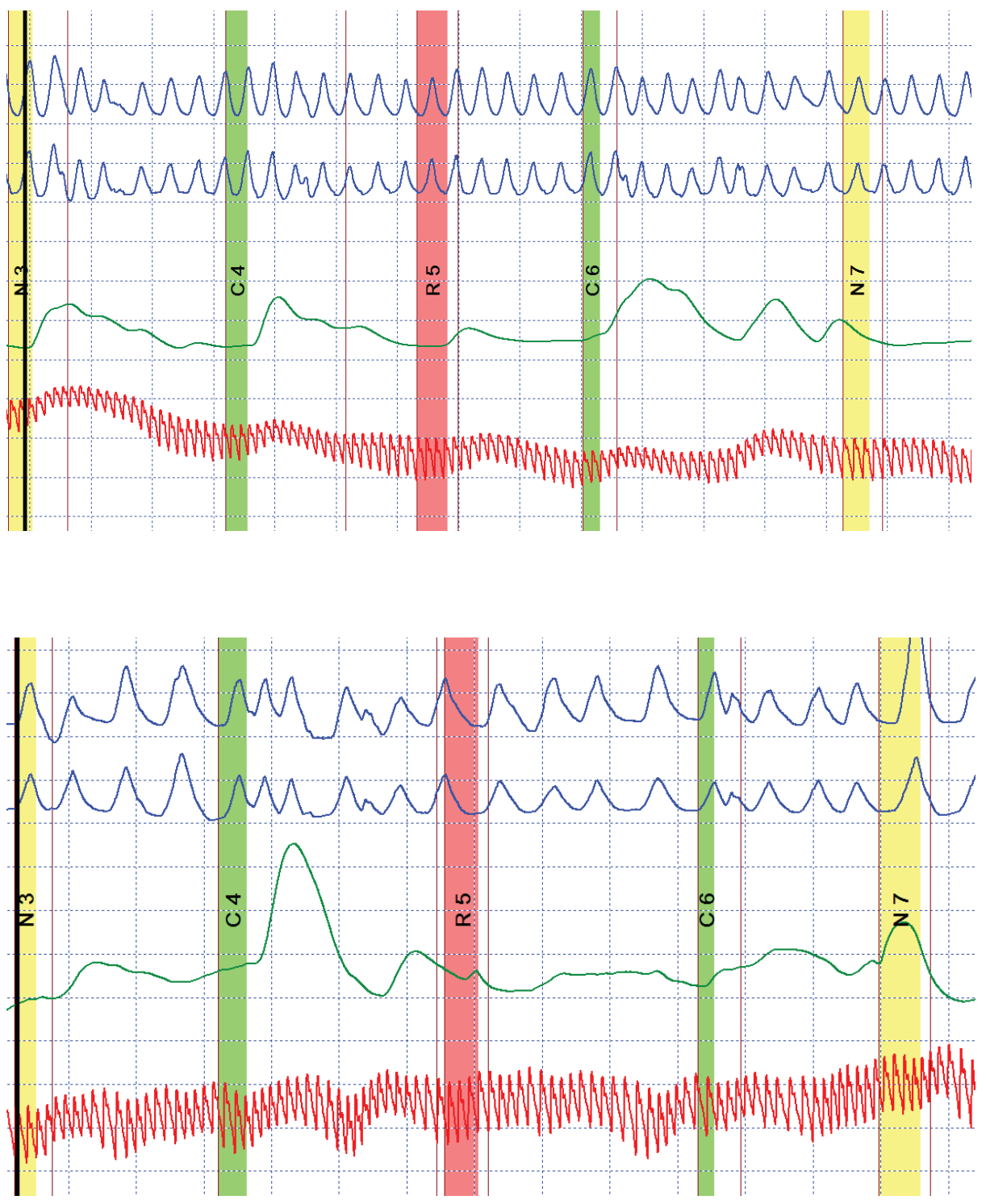


\section{Conclusions}

The PVT clearly helps the examiner to reconfirm his/her results and support the initial finding with something objective that is not related to the efficiency of comparison question. In addition, in cases where there is suspicion of deliberate distortions, they can easily be confirmed with the use of the PVT.

\section{Abstract}

This paper involves fifty-one re-examinations of original polygraph tests that resulted in conflicted outcomes and examinations where deliberate distortions were believed to have been employed. The Polygraph Validation Test (PVT) was successfully employed in these re-examinations to rectify the original problems and/or confirm attempts by examinees at countermeasures or augmentations.

\section{References}

Gordon N., Cochetti P. The Horizontal Scoring System. Polygraph 1987, 16, 2.

Gordon N., Fleisher W. Academy for Scientific Investigative Training's Horizontal Scoring System and Examiner's Algorithm for Chart Interpretation. Polygraph 1999, 28, 1.

Gordon N., Fleisher W. Effectiveness of the Integrated Zone Comparison Technique (IZCT) with Various Scoring Systems in a Mock Crime Experiment by Students. European Polygraph 2012, 6, 1 (19).

Gordon N., Fleisher W., et al. A Field Study of the Integrated Zone Comparison Technique. Polygraph 2000, 29, 3.

Gordon N., Mohammed F., Farro S., et al. Integrated Zone Comparison Polygraph Technique Accuracy with Scoring Algorithms. Physiology and Behavior 2006, 87.

Shurany T., Chaves F. The Integrated Zone Comparison Technique and ASIT PolySuite Algorithm: A Field Validity Study. European Polygraph 2010, 4, 2 (12).

Shurany T. Polygraph Verification Test. European Polygraph 2011, 5, 2 (16). 
